PROCEEDINGS OF THE

AMERICAN MATHEMATICAL SOCIETY

Volume 125, Number 6, June 1997, Pages 1593-1605

S 0002-9939(97)03752-0

\title{
GROWTH OF GRADED NOETHERIAN RINGS
}

\author{
DARIN R. STEPHENSON AND JAMES J. ZHANG \\ (Communicated by Lance W. Small)
}

\begin{abstract}
We show that every graded locally finite right noetherian algebra has sub-exponential growth. As a consequence, every noetherian algebra with exponential growth has no finite dimensional filtration which leads to a right (or left) noetherian associated graded algebra. We also prove that every connected graded right noetherian algebra with finite global dimension has finite GK-dimension. Using this, we can classify all connected graded noetherian algebras of global dimension two.
\end{abstract}

\section{INTRODUCTION}

The study of graded algebras has gained prominence recently due to developments in the area of noncommutative projective geometry (for example, see [ATV]). In this article, we prove several results involving the growth of graded algebras, and give applications to the theory of regular algebras.

Throughout, $k$ is a field and all algebras and modules are over the base field $k$. The term 'algebra' will be used to denote an associative $k$-algebra with unity. Let $R$ be an (ungraded) algebra and $M$ a right $R$-module. The Gelfand-Kirillov dimension of $M$ is defined by

$$
\operatorname{GKdim}(M)=\varlimsup_{V, N} \varlimsup_{n \rightarrow \infty} \log _{n}\left(\operatorname{dim}_{k}\left(N V^{n}\right)\right)
$$

where $V \subset R$ and $N \subset M$ run over all finite dimensional subspaces. We say $M$ has exponential growth if

$$
\varlimsup_{V, N} \varlimsup_{n \rightarrow \infty}\left(\operatorname{dim}_{k}\left(N V^{n}\right)\right)^{\frac{1}{n}}>1
$$

where $V$ and $N$ are as above. Otherwise, we say $M$ has sub-exponential growth. If $\operatorname{GKdim}(M)<\infty$, then $M$ has sub-exponential growth, but the converse is not true in general (see [S2]). In this paper we are interested in $\mathbb{N}$-graded algebras $A=\oplus_{i \geq 0} A_{i}$. If $A_{0}=k$, then $A$ is called connected graded. A graded module $M=\oplus_{i \in \mathbb{Z}} M_{i}$ is called locally finite if $\operatorname{dim}_{k}\left(M_{i}\right)<\infty$ for all $i$. In particular, if $A$ is a graded right noetherian algebra and $A_{0}$ is finite dimensional, then $A$ is locally finite.

Our main result is the following theorem.

Received by the editors December 5, 1995.

1991 Mathematics Subject Classification. Primary 16P90, 16W50, 16 E10.

Key words and phrases. Sub-exponential growth, GK-dimension, graded ring, global dimension.

The second author was supported by the NSF. 
Theorem 0.1. If $A$ is a graded locally finite right noetherian algebra, then $A$ has sub-exponential growth.

The proof of this theorem is given in Section 1. It involves showing that, if $A$ has exponential growth, then there exists an increasing sequence of natural numbers $\left\{l_{i}: i \geq 1\right\}$ such that $\operatorname{dim}_{k} A_{l_{n}}>\sum_{i=1}^{n-1} \operatorname{dim}_{k} A_{l_{n}-l_{i}}$ for all $n$. This fact is then used to construct an infinite ascending chain of right ideals in $A$.

Theorem 0.1 can be used to answer a question of J. C. McConnell and J. C. Robson [MR, 8.3.10].

Proposition 0.2. There is a finitely generated noetherian algebra for which no finite dimensional filtration leads to a right noetherian associated graded ring.

In particular, an easy corollary of Theorem 0.1 shows that the desired example is given by any finitely generated noetherian ungraded algebra with exponential growth.

In Section 2, we use Theorem 0.1 to prove the following result.

Theorem 0.3. If $A$ is a connected graded right noetherian algebra with finite global dimension, then $A$ has finite GK-dimension.

Theorem 0.3 is then used to answer a question about regular algebras. Let $k_{A}$ be the trivial module $A / \oplus_{i>0} A_{i}$. A connected graded algebra of global dimension $d$ will be called regular if $\operatorname{Ext}_{A}^{i}\left(k_{A}, A\right)=0$ if $i \neq d$ and $\operatorname{dim}_{k}\left(\operatorname{Ext}_{A}^{d}\left(k_{A}, A\right)\right)=1$. This is different from the definition in $[\mathrm{AS}]$ in that we omit the condition $\operatorname{GKdim}(A)<\infty$. If $A$ is noetherian, then by Theorem $0.3, A$ automatically has finite GK-dimension.

In Section 3, we prove the following.

Theorem 0.4. If $A$ is a connected graded noetherian algebra of global dimension two, then $A$ is regular.

For completeness, we also include a classification of noetherian regular algebras of global dimension two (Proposition 3.3). Finally, we give an example of a non-noetherian regular algebra of global dimension two with exponential growth (Example 3.4).

\section{NOETHERIAN GRADED RINGS}

If $A$ is a graded locally finite right noetherian algebra and $M=\oplus_{i=q}^{\infty} M_{i}$ is a noetherian graded right $A$-module, then

$$
\operatorname{GKdim}(M)=\varlimsup_{n \rightarrow \infty} \log _{n}\left(\sum_{i \leq n} \operatorname{dim}_{k}\left(M_{i}\right)\right) .
$$

It is easy to see that a noetherian graded right $A$-module $M$ has exponential growth if and only if $\varlimsup_{n \rightarrow \infty}\left(\sum_{i \leq n} \operatorname{dim}_{k}\left(M_{i}\right)\right)^{\frac{1}{n}}>1$.

Lemma 1.1. Let $\left\{d_{n} \mid n \in \mathbb{N}\right\}$ be a sequence of non-negative integers with infinitely many nonzero $d_{n}$ 's.

1. $\varlimsup_{n \rightarrow \infty}\left(\sum_{i \leq n} d_{i}\right)^{\frac{1}{n}}=\varlimsup_{n \rightarrow \infty} d_{n}^{\frac{1}{n}}$.

2. Suppose $d_{n}>0$ for all $n \in \mathbb{N}$. Given positive integers $l_{0}, l_{1}, \cdots, l_{s}$,

$$
\varlimsup_{n \rightarrow \infty} \min \left\{\left(d_{n} / d_{n-l_{j}}\right)^{\frac{1}{l_{j}}} \mid j=0,1, \cdots, s\right\} \geq \varlimsup_{n \rightarrow \infty} d_{n}^{\frac{1}{n}} .
$$


3. If $\varlimsup_{n \rightarrow \infty} d_{n}^{\frac{1}{n}}>1$, then there is a subsequence $\left\{l_{i} \mid i \in \mathbb{N}\right\} \subset \mathbb{N}$ such that $d_{l_{0}}>1$ and $d_{l_{s}}>\sum_{i<s}^{n \rightarrow \infty} d_{l_{s}-l_{i}}$ for all $s \geq 1$.

Proof. 1. It is trivial that $\varlimsup_{n \rightarrow \infty} d_{n}^{\frac{1}{n}} \leq \varlimsup_{n \rightarrow \infty}\left(\sum_{i \leq n} d_{i}\right)^{\frac{1}{n}}$. Since there are infinitely many nonzero $d_{n}$ 's, $\varlimsup_{n \rightarrow \infty} d_{n}^{\frac{1}{n} \rightarrow \infty} \geq 1$. Suppose $\varlimsup_{n \rightarrow \infty} d_{n}^{\frac{1}{n}}<\alpha$ for some $\alpha>1$. Then $d_{n}<\alpha^{n}$ for all $n \geq n_{0}$. Hence $\sum_{i \leq n} d_{i}<\left(\sum_{i \leq n_{0}}^{n \rightarrow \infty} d_{i}\right)+n \alpha^{n}$ for all $n$. Therefore $\varlimsup_{n \rightarrow \infty}\left(\sum_{i \leq n} d_{n}\right)^{\frac{1}{n}} \leq \alpha$. This implies that $\varlimsup_{n \rightarrow \infty}\left(\sum_{i \leq n} d_{n}\right)^{\frac{1}{n}} \leq \varlimsup_{n \rightarrow \infty} d_{n}^{\frac{1}{n}}$.

2. If $\varlimsup_{n \rightarrow \infty} \min \left\{\left(d_{n} / d_{n-l_{j}}\right)^{\frac{1}{l_{j}}} \mid j=0,1, \cdots, s\right\}<\alpha$ for some $\alpha$, we need to show $\varlimsup_{n \rightarrow \infty} d_{n}^{\frac{1}{n}} \leq \alpha$. There exists $m_{0}$ such that $\min \left\{\left(d_{n} / d_{n-l_{j}}\right)^{\frac{1}{l_{j}}} \mid j=0,1, \cdots, s\right\}<\alpha$ for all $n \geq m_{0}$. Hence for some $i$, we have $d_{n}<\alpha^{l_{i}} d_{n-l_{i}}$. By induction we have $d_{n}<\alpha^{n-m_{0}} \bar{d}_{m_{0}}$, where $\bar{d}_{m_{0}}=\max \left\{d_{i} \alpha^{m_{0}-i} \mid i=0, \cdots, m_{0}\right\}$. Therefore $\varlimsup_{n \rightarrow \infty} d_{n}^{\frac{1}{n}} \leq \alpha$.

3. We may assume $d_{n}>0$ by replacing 0 by 1 whenever $d_{n}=0$. The conclusion will not be changed because for all $s, d_{l_{s}}>1$. Suppose $\varlimsup_{n \rightarrow \infty} d_{n}^{\frac{1}{n}}=a>1$. Choose $b$ with $1<b<a$. Let $l_{0}$ be a positive integer such that $d_{l_{0}}>1$ and $b^{l_{0}}>2$. Construct $l_{i}$ inductively as follows. Suppose we have obtained natural numbers $l_{0}<l_{1}<\cdots<l_{s}$ such that $d_{l_{j}}>\sum_{i<j} d_{l_{j}-l_{i}}$ and $b^{l_{j}}>2^{j+1}$ for all $j \geq 1$. By part 2, $\varlimsup_{n \rightarrow \infty} \min \left\{\left(d_{n} / d_{n-l_{j}}\right)^{\frac{1}{l_{j}}} \mid j=0,1, \cdots, s\right\} \geq a>b$. Hence there is an infinite subsequence $\left\{n_{i}\right\} \subset \mathbb{N}$ such that

$$
\min \left\{\left(d_{n_{i}} / d_{n_{i}-l_{j}}\right)^{\frac{1}{l_{j}}} \mid j=0,1, \cdots, s\right\}>b .
$$

Let $l_{s+1}$ be in the subsequence $\left\{n_{i}\right\}$ such that $l_{s+1}>l_{s}$ and $b^{l_{s+1}}>2^{s+2}$. Then by $(1-1)$

$$
\sum_{i<s+1} d_{l_{s+1}-l_{i}}<\sum_{i<s+1} d_{l_{s+1}} b^{-l_{i}}=d_{l_{s+1}} \sum_{i<s+1} b^{-l_{i}}<d_{l_{s+1}}\left(\sum_{i \geq 0} 2^{-(i+1)}\right)=d_{l_{s+1}} .
$$

Theorem 1.2. If $A$ is a graded locally finite right noetherian algebra, then $A$ has sub-exponential growth. As a consequence, every graded noetherian right $A$-module has sub-exponential growth.

Proof. Let $d_{n}=\operatorname{dim}_{k}\left(A_{n}\right)$. If $A$ has exponential growth, by Lemma 1.1.1, $\varlimsup_{n \rightarrow \infty} d_{n}^{\frac{1}{n}}=$ $a>1$. By Lemma 1.1.3, there is a sequence of $\left\{l_{i} \mid i \in \mathbb{N}\right\}$ such that $d_{l_{0}}>1$ and $d_{l_{s}}>\sum_{i<s} d_{l_{s}-l_{i}}$ for all $s$. Hence for this subsequence, $\operatorname{dim}\left(A_{l_{i}}\right)=d_{l_{i}}>1$. We define inductively a sequence of right ideals $I^{s}=\sum_{i \leq s} x_{i} A$, for some $x_{i} \in A_{l_{i}}$, as follows. Suppose we have obtained $x_{i} \in A_{l_{i}}$ for all $i<s$. Since $d_{l_{s}}>\sum_{i<s} d_{l_{s}-l_{i}}$, we have

$$
\operatorname{dim}\left(A_{l_{s}}\right)>\sum_{i<s} \operatorname{dim}\left(A_{l_{s}-l_{i}}\right) \geq \sum_{i<s} \operatorname{dim}\left(\left(x_{i} A\right)_{l_{s}}\right) \geq \operatorname{dim}\left(I_{l_{s}}^{s-1}\right) .
$$

Pick $x_{s}$ in $A_{l_{s}}$ but not in $I^{s-1}$, and form $I^{s}=x_{s} A+I^{s-1}$. Hence $I^{s-1} \neq I^{s}$ and $\left\{I^{s} \subset A \mid s \in \mathbb{N}\right\}$ is a strictly ascending chain of right ideals. As $A$ is right noetherian, this is a contradiction. Therefore $A$ has sub-exponential growth. If 
$A$ has sub-exponential growth, then every finitely generated $A$-module has subexponential growth.

Remark. Using Theorem 1.2, it is easy to show that any $\mathbb{Z}$-graded locally finite right noetherian algebra has sub-exponential growth. If $\oplus_{i \in \mathbb{Z}} A_{i}$ is such an algebra, [NV, A.II.3.4] shows that the $\mathbb{N}$-graded rings $B=\oplus_{i \geq 0} A_{i}$ and $C=\oplus_{i \leq 0} A_{i}$ are right noetherian. Thus by Theorem 1.2, $B$ and $C$ have sub-exponential growth, and the result follows easily for $A$.

Using Theorem 1.2 we can answer a question asked by McConnell and Robson in [MR, 8.3.10].

Corollary 1.3. Let $R$ be a finitely generated right noetherian algebra with exponential growth and let $F:=\left\{F_{n} \mid n \in \mathbb{N}\right\}$ be a finite dimensional filtration of $R$. Then the associated graded ring $\operatorname{gr}_{F} R$ is neither left nor right noetherian.

Proof. Since $R$ is finitely generated, $F_{m}$ generates $R$ for some $m$. Since $R$ has exponential growth, $\varlimsup_{n \rightarrow \infty}\left(\operatorname{dim}_{k}\left(F_{m}^{n}\right)\right)^{\frac{1}{n}}>1$. This implies that $\varlimsup_{n \rightarrow \infty}\left(\operatorname{dim}_{k}\left(F_{n}\right)\right)^{\frac{1}{n}}>$ 1 because $F_{m}^{n} \subset F_{m n}$. Hence $\operatorname{gr}_{F} R$ has exponential growth and by Theorem 1.2, $\operatorname{gr}_{F} R$ is not noetherian.

There are well-known examples of finitely generated noetherian rings with exponential growth, such as group rings of finitely generated polycyclic-by-finite groups which are not nilpotent-by-finite [KL, 11.9]. The next example is due to M. Smith [S1].

Example 1.4. There is a finitely generated noetherian algebra which has exponential growth.

Let $k[x, y]$ be the commutative polynomial ring in two variables. Let $\sigma$ be the automorphism of $k[x, y]$ defined by $\sigma(x)=y+x^{2}$ and $\sigma(y)=x$. Define $R$ to be the skew polynomial extension $k[x, y]\left[z, z^{-1}, \sigma\right]$. Hence $R$ is a noetherian algebra generated by $x, y, z, z^{-1}$. It is easy to see that $\operatorname{deg}\left(\sigma^{n}(x)\right)=2^{n}$. By $[\mathrm{S} 1,10], R$ has exponential growth. By Corollary 1.3, no finite dimensional filtration of $R$ leads to a right (or left) noetherian associated graded ring. The above results show that the noetherian condition is more restrictive on the growth of graded rings than on the growth of ungraded rings.

Remark. 1. J. T. Stafford has pointed out that the ring $S$ defined in [MS, Section 3] may also give an answer to the question in [MR, 8.3.10]. In that paper, it is shown that $\operatorname{gr}_{F} S$ is non-noetherian for every appropriately nice filtration $F$ (see [MS, 2.3]). It is unknown whether $\operatorname{gr}_{F} S$ is non-noetherian for all finite dimensional filtrations. If so, this example would give an answer to [MR, 8.3.10] with the added feature that $\operatorname{GKdim}(S)<\infty$.

2. It is unknown if every connected graded right noetherian algebra with subexponential growth has finite GK-dimension. The following examples show that if we omit either the noetherian condition or the graded condition, sub-exponential growth does not imply finite GK-dimension. Example 1.5 is due to M. Smith [S2], and Example 1.6 is work of R. Resco and L. Small [RS].

Example 1.5. There is a finitely generated connected graded algebra $U$ such that $U$ has sub-exponential growth and $\operatorname{GKdim}(U)=\infty$. 
Let $L$ be the infinite dimensional Lie algebra with basis $x, y_{1}, y_{2}, \ldots$ subject to the relations $\left[x, y_{i}\right]=y_{i+1},\left[y_{i}, y_{j}\right]=0$. Then the universal enveloping algebra $U=U(L)$ has sub-exponential growth and infinite GK-dimension (see [S2, 8]). Let $\operatorname{deg}(x)=1$ and $\operatorname{deg}\left(y_{i}\right)=i$. Then $U$ is a connected graded ring generated by $x$ and $y_{1}$, and has infinitely many defining relations.

Example 1.6. There is a finitely generated noetherian (ungraded) algebra $R$ such that $R$ has sub-exponential growth and $\operatorname{GKdim}(R)=\infty$.

Let $\operatorname{char}(k)=p>0$, and let $U$ be as in Example 1.5. The center $C$ of $U$ is $k\left[y_{i}^{p}\right]$. Let $K$ be the field of fractions of $C$, and let $R$ be the $K$-algebra $U \otimes_{C} K$. By [RS, Theorem 1], $R$ is noetherian and is generated as a $K$-algebra by $x$ and $y_{1}$. By Example 1.5, $R$ has sub-exponential growth and by [RS, p. 551], $\operatorname{GKdim}(R)=\infty$.

\section{Graded Rings With Finite GLOBAL Dimension}

A graded module $M$ is called left bounded if $M_{i}=0$ for all $i \ll 0$. Recall that the Hilbert series of a graded left bounded locally finite module $M=\oplus_{i \geq s} M_{i}$ is defined to be the formal power series

$$
h_{M}(t)=\sum_{n} \operatorname{dim}_{k}\left(M_{n}\right) t^{n} \in \mathbb{Z}\left[\left[t, t^{-1}\right]\right] .
$$

For a graded module $M$, the degree shift $s(M)$ is defined by $s(M)_{n}=M_{n+1}$ and $s^{l}(M)$ is denoted by $M(l)$ for all $l \in \mathbb{Z}$. It is easy to see that $h_{M(l)}(t)=t^{-l} h_{M}(t)$ for all $l$.

Lemma 2.1. Let $p(t)$ and $q(t)$ be relatively prime polynomials such that $p(t)=$ $\prod_{i=1}^{l}\left(1-r_{i} t\right)$. Suppose $q(t) / p(t)=\sum_{n \geq 0} d_{n} t^{n}$ and $\varlimsup_{n \rightarrow \infty}\left|d_{n}\right|^{\frac{1}{n}} \leq 1$. Then $\left|r_{i}\right| \leq 1$ for all $i$.

Proof. Since $\varlimsup_{n \rightarrow \infty}\left|d_{n}\right|^{\frac{1}{n}} \leq 1, \sum_{n \geq 0} d_{n} t^{n}$ has convergence radius at least 1 . Hence $\sum_{n \geq 0} d_{n} t^{n}=q(t) / p(t)$ converges for all $|t|<1$. Thus $p(t)=\prod_{i}\left(1-r_{i} t\right) \neq 0$ if $|t|<1$. Therefore $\left|r_{i}\right| \leq 1$.

Let $f(n)$ be a function from $\mathbb{Z}$ to $\mathbb{N}$. If there exist an integer $w$ and polynomial functions $f_{1}(n), \cdots, f_{w}(n) \in \mathbb{Q}[n]$ such that $f(n)=f_{s}(n)$ for all $n \equiv s(\bmod w)$, then $f(n)$ is called a multi-polynomial function. Define

$$
\operatorname{deg}(f(n))=\max \left\{\operatorname{deg}\left(f_{s}(n)\right) \mid s=1, \cdots, w\right\} .
$$

For any polynomial $p(t)$, let $m(p(t))$ be the multiplicity of 1 as a root.

Corollary 2.2. Let $M$ be a graded left bounded locally finite right $A$-module with sub-exponential growth. Suppose $h_{M}(t)=t^{l} q(t) / p(t)$ and $p(t)$ and $q(t)$ are relatively prime polynomials with integer coefficients. If $p(0)=1$, then every root of $p(t)$ is a root of unity and $\mathrm{GKdim}(M)=m(p(t))<\infty$.

Proof. Let $p(t)=\prod_{i}\left(1-r_{i} t\right)$. By Lemma 2.1, each $r_{i}$ has absolute value at most 1. Since $p(t)$ has integer coefficients, the product of all the $r_{i}$ 's is an integer which has absolute value at least 1 . Hence every $r_{i}$ has absolute value 1 . The roots of $p(t)$ are $\left\{1 / r_{i}\right\}$. Hence each $1 / r_{i}$ is an algebraic integer with absolute value 1 , whence it is a root of 1 (see for example [CF, Lemma 2, p. 72]). As a consequence, each $r_{i}$ is a root of 1 . Expanding $t^{l} q(t) / p(t)$ as a Taylor series $\sum_{n \geq l} d_{n} t^{n}$ gives that $d_{n}=\operatorname{dim} M_{n}$ is a multi-polynomial function of $n$ for $n \gg 0$ [St, 4.1]. If $M$ is finite 
dimensional over $k$, then $p(t)=1$ and $\operatorname{GKdim}(M)=0$. Now we assume that $M$ is infinite dimensional over $k$ and $p(t)$ and $q(t)$ are relatively prime. By [ATV, 2.21(i)], $p(1)=0$. By [ATV, 2.21(ii)], $d_{n}$ is a multi-polynomial function of $n$ of degree $m(p(t))-1$ (for $n \gg 0)$. Consequently, $\operatorname{GKdim}(M)=m(p(t))<\infty$.

Let $A$ be a connected graded (not necessarily locally finite) algebra of finite global dimension $d$. Every graded left bounded projective $A$-module is free, and thus is a sum of shifts of $A$. For every graded left bounded $A$-module $M$, we have an augmented minimal free resolution of $M$

$$
0 \longrightarrow \oplus_{i=1}^{z_{d}} A\left(-l_{i}^{d}\right) \longrightarrow \cdots \longrightarrow \oplus_{i=1}^{z_{1}} A\left(-l_{i}^{1}\right) \longrightarrow \oplus_{i=1}^{z_{0}} A\left(-l_{i}^{0}\right) \longrightarrow M \longrightarrow 0
$$

where $z_{i}$ is allowed to be infinite (even uncountably infinite). A minimal free resolution of $M$ is the complex (2-1) without $M$. We say $M$ has a finite minimal free resolution if each $z_{i}$ is finite. If $M$ has a finite minimal free resolution of the form (21 ), the characteristic polynomial of $M$ is defined as $c_{M}(t)=\sum_{j=0}^{d}(-1)^{j}\left(\sum_{i=1}^{z_{j}} t^{l_{i}^{j}}\right)$.

If $M$ and $N$ are graded $A$-modules, we use $\operatorname{Hom}_{A}^{d}(M, N)$ to denote the set of all $A$-module homomorphisms $h: M \rightarrow N$ such that $h\left(M_{i}\right) \subseteq N_{i+d}$. We set $\underline{\operatorname{Hom}}_{A}(M, N)=\oplus_{d \in \mathbb{Z}} \operatorname{Hom}_{A}^{d}(M, N)$, and we denote the corresponding derived functors by $\underline{\operatorname{Ext}}_{A}^{i}(M, N)$. If $M$ has a finite free resolution, $\underline{\operatorname{Ext}}_{A}^{i}(M, N)=\operatorname{Ext}_{A}^{i}(M, N)$, but this is not true in general. If $M$ is a graded right module and $N$ is a graded left module, the groups $\operatorname{Tor}_{i}^{A}(M, N)$ have a graded structure, which we denote by $\underline{\operatorname{Tor}}_{i}^{A}(M, N)$. Note that $A$ has finite right global dimension if and only if $A$ has finite left global dimension and

$$
\text { right. gl. } \operatorname{dim}(A)=\max \left\{i \mid \underline{\operatorname{Tor}}_{i}^{A}\left(k_{A},{ }_{A} k\right) \neq 0\right\}=\text { left. gl. } \operatorname{dim}(A)
$$

Lemma 2.3. Suppose $M$ has a finite free resolution of the form (2-1) and let $c_{M}(t)$ be the characteristic polynomial of $M$. Then $h_{M}(t)=c_{M}(t) h_{A}(t)$ and $\sum_{N}(-1)^{i} h_{\underline{\operatorname{Ext}}^{i}(M, N)}(t)=c_{M}\left(t^{-1}\right) h_{N}(t)$ for all left bounded locally finite A-modules $\sum_{i}$

Proof. Given any complex of graded left bounded locally finite $k$-modules

$$
M^{\bullet}:=0 \longrightarrow M_{n} \longrightarrow M_{n-1} \longrightarrow \cdots \longrightarrow M_{1} \longrightarrow M_{0} \longrightarrow 0
$$

by additivity of $\operatorname{dim}_{k}(-)$, we have

$$
\sum_{i}(-1)^{i} h_{M_{i}}(t)=\sum_{i}(-1)^{i} h_{H^{i}(M \bullet)}(t)
$$

where $H^{i}\left(M^{\bullet}\right)$ is the $i$-th homology of $M^{\bullet}$ for all $i$. Applying (2-2) to (2-1), we have $h_{M}(t)=c_{M}(t) h_{A}(t)$. To compute $\underline{\operatorname{Ext}^{i}}(M, N)$, we apply $\underline{\operatorname{Hom}}(-, N)$ to the free resolution of $M$ and obtain

$$
0 \longleftarrow \oplus_{i=1}^{z_{d}} N\left(l_{i}^{d}\right) \longleftarrow \cdots \longleftarrow \oplus_{i=1}^{z_{1}} N\left(l_{i}^{1}\right) \longleftarrow \oplus_{i=1}^{z_{0}} N\left(l_{i}^{0}\right) \longleftarrow 0
$$

Then it follows from (2-2) and (2-3) that $\sum_{i}(-1)^{i} h_{\underline{\operatorname{Ext}}^{i}(M, N)}(t)=c_{M}\left(t^{-1}\right) h_{N}(t)$.

The augmented minimal free resolution of the module $k_{A}:=A /\left(\oplus_{i>0} A_{i}\right)$ is of the form

$$
0 \longrightarrow \oplus_{i=1}^{z_{d}} A\left(-s_{i}^{d}\right) \longrightarrow \cdots \longrightarrow \oplus_{i=1}^{z_{1}} A\left(-s_{i}^{1}\right) \longrightarrow A \longrightarrow k_{A} \longrightarrow 0 .
$$

Since $A$ is connected graded and (2-4) is minimal, all $s_{i}^{j}$ are positive. The characteristic polynomial of $k_{A}$ is denoted by $p_{A}(t)$, and $p_{A}(t)=1+\sum_{j=1}^{n}(-1)^{j}\left(\sum_{i=1}^{z_{j}} t^{s_{i}^{j}}\right)$. 
Consequently, $p_{A}(0)=1$. Since $h_{k_{A}}(t)=1$, we have $p_{A}(t) h_{A}(t)=1$ [ATV, 2.9]. For a graded right $A$-module $M, h_{M}(t)=c_{M}(t) / p_{A}(t)$.

The next theorem is an analogue of [ATV, 2.14].

Theorem 2.4. Let $A$ be a connected graded right noetherian algebra with finite global dimension.

1. A has finite GK-dimension.

2. $p_{A}\left(t^{-1}\right)= \pm t^{-l} p_{A}(t)$ and $h_{A}\left(t^{-1}\right)= \pm t^{l} h_{A}(t)$, where $l=\operatorname{deg}\left(p_{A}(t)\right)$. In particular the leading coefficient of $p_{A}(t)$ is 1 or -1 .

3. GK-dimension is finitely partitive on right A-modules. As a consequence, $\operatorname{Kdim}(M) \leq \operatorname{GKdim}(M)$ for all noetherian right $A$-modules $M$.

Proof. 1 and 2. By Theorem 1.2, $A$ has sub-exponential growth. Suppose $p(t)=$ $\prod_{i}\left(1-r_{i} t\right)$. By Corollary 2.2, each $r_{i}$ is a root of 1 . Hence $r_{i}^{-1}=\bar{r}_{i}$. Since $p(t)$ has integer coefficients, the product of all $r_{i}$ is either 1 or -1 . Hence

$$
t^{l} p\left(t^{-1}\right)=\prod_{i}\left(t-r_{i}\right)= \pm \prod_{i}\left(1-r_{i}^{-1} t\right)= \pm \prod_{i}\left(1-\overline{r_{i}} t\right)=\overline{ \pm p(t)}= \pm p(t) .
$$

Thus we have proved 2. Let $m\left(p_{A}(t)\right)$ be the multiplicity of 1 as a root of $p_{A}(t)$. By Corollary 2.2, GKdim $(A)=m\left(p_{A}(t)\right)<\infty$.

3. Let $p_{A}(t)=(1-t)^{m} p_{0}(t)$ where $m=m\left(p_{A}(t)\right)$ and $p_{0}(1) \neq 0$. Since $p_{A}(0)=1$ and $p_{A}(t) \neq 0$ for all $t<1$, we have $p_{0}(1)>0$ by the Intermediate Value Theorem. Hence $p_{0}(1)$ is a positive integer. For every graded right $A$-module $M$ with $\operatorname{GKdim}(M)=g$,

$$
h_{M}(t)=c_{M}(t) / p_{A}(t)=f(t) /(1-t)^{g} p_{0}(t)
$$

where $f(t)$ is a polynomial function with integer coefficients and $f(1) \neq 0$. Since $M$ is nonzero, $\lim _{t \rightarrow 1^{-}} h_{M}(t)>0$. Hence $f(1) / p_{0}(1)>0$ and consequently $f(1)$ is a positive integer. Define the multiplicity of $M$ to be $f(1)$ (in [ATV, 2.21(iii)] the multiplicity of $M$ is defined to be $\left.f(1) / p_{0}(1)\right)$. Since Hilbert series is additive on exact sequences, the multiplicity is additive. By [MR, 8.4.8 and 8.4.9], GKdimension is finitely right partitive and $\operatorname{Kdim}(M) \leq \operatorname{GKdim}(M)$.

The following is a consequence of Lemma 2.3 and Theorem 2.4.2.

Corollary 2.5. Let $A$ be a connected graded right noetherian algebra of global dimension d. If $\underline{\operatorname{Ext}}^{i}\left(k_{A}, A\right)=0$ for all $i \neq d$, then $\underline{\operatorname{Ext}}^{d}\left(k_{A}, A\right)=k(l)$ for some integer $l$.

Proof. By Theorem 2.4, $p_{A}\left(t^{-1}\right)= \pm t^{-l} p(t)$ for some $l$. Since $A$ is right noetherian, $k_{A}$ has finite free resolution. Since $\underline{\operatorname{Ext}}^{i}\left(k_{A}, A\right)=0$ for all $i \neq d$, by Lemma 2.3,

$$
\begin{aligned}
(-1)^{d} h_{\underline{\operatorname{Ext}}^{d}\left(k_{A}, A\right)}(t) & =\sum_{i}(-1)^{i} h_{\underline{\operatorname{Ext}}^{i}\left(k_{A}, A\right)}(t) \\
& =c_{k_{A}}\left(t^{-1}\right) h_{A}(t)=p_{A}\left(t^{-1}\right) / p_{A}(t)= \pm t^{-l} .
\end{aligned}
$$

Hence $\underline{\operatorname{Ext}}^{d}\left(k_{A}, A\right)=k(l)$.

\section{Graded Regular algebras}

Let $A$ be a connected graded (not necessarily locally finite) algebra of global dimension $d$. Recall that $A$ is regular if $\operatorname{Ext}_{A}^{i}\left(k_{A}, A\right)=0$ for all $i \neq d$, and $\operatorname{Ext}_{A}^{d}\left(k_{A}, A\right)$ is one dimensional (in some papers, such algebras are called Artin-Schelter regular). 
If $A$ is also assumed to be noetherian, then $\operatorname{GKdim}(A)<\infty$ by Theorem 2.4. On the other hand we will see that there exist non-noetherian regular algebras with exponential growth [Example 3.4].

Some nice properties of injective resolutions of Gorenstein rings are given in $[\mathrm{Zh}$, $0.3]$. The next proposition shows some nice properties of free resolutions of $k$ for regular rings.

Proposition 3.1. Let $A$ be a connected graded algebra of global dimension d. Suppose that $\operatorname{Ext}^{i}\left(k_{A}, A\right)=0$ for all $i \neq d$ and $\operatorname{Ext}^{d}\left(k_{A}, A\right)$ is finite dimensional. Then

1. $k_{A}$ and ${ }_{A} k$ have finite free resolutions. In particular, $A$ is finitely presented and locally finite. Also, $\underline{\operatorname{Ext}}_{A}^{i}\left(k_{A}, A\right)=\operatorname{Ext}_{A}^{i}\left(k_{A}, A\right)$ and $\operatorname{Ext}_{A}^{i}\left({ }_{A} k, A\right)=$ $\operatorname{Ext}_{A}^{i}\left({ }_{A} k, A\right)$ for all $i$.

2. $\underline{\operatorname{Ext}}_{A}^{i}\left({ }_{A} k, A\right)=0$ for all $i \neq d$ and $\underline{\operatorname{Ext}}_{A}^{d}\left({ }_{A} k, A\right)=\underline{\operatorname{Ext}}_{A}^{d}\left(k_{A}, A\right)=k(e)$ for some e. In particular, $A$ is regular.

3. The d-th term of the minimal free resolution of $k_{A}\left(\right.$ or $\left.{ }_{A} k\right)$ is $A(-e)$, and in the notation of $(2-4), 0<s_{i}^{j}<e$ for all $0<j<d$ and all $i$.

4. $t^{l} p_{A}\left(t^{-1}\right)=(-1)^{d} p_{A}(t)$ where $l=\operatorname{deg}\left(p_{A}(t)\right)$. Furthermore, $e=l$ and $d \leq e$.

5. If $d=e$, then $s_{i}^{j}=j$ for all $j$ and $i$.

6. If $A$ has sub-exponential growth, then $\operatorname{GKdim}(A) \leq e$.

Proof. Consider the minimal augmented free resolution of $k_{A}$,

$$
0 \longrightarrow \oplus_{i=1}^{z_{d}} A\left(-s_{i}^{d}\right) \longrightarrow \cdots \longrightarrow \oplus_{i=1}^{z_{1}} A\left(-s_{i}^{1}\right) \longrightarrow A \longrightarrow k_{A} \longrightarrow 0
$$

where $z_{i}$ is allowed to be infinite (even uncountable). We will first show that each $z_{i}$ is finite. Since (3-1) is minimal, every boundary map $\partial_{j}$ from $\oplus_{i=1}^{z_{j}} A\left(-s_{i}^{j}\right)$ to $\oplus_{i=1}^{z_{j-1}} A\left(-s_{i}^{j-1}\right)$ can be represented by a matrix whose entries are in $A_{>0}:=$ $\sum_{i>0} A_{i}$. Consequently,

$$
\min \left\{s_{i}^{j} \mid 1 \leq i \leq z_{j}\right\}<\min \left\{s_{i}^{j+1} \mid 1 \leq i \leq z_{j+1}\right\} .
$$

Applying $\operatorname{Hom}_{A}(-, A)$ to the deleted resolution of (3-1) gives the complex

$$
0 \longleftarrow \prod_{i=1}^{z_{d}} A\left(s_{i}^{d}\right) \longleftarrow \cdots \longleftarrow \prod_{i=1}^{z_{1}} A\left(s_{i}^{1}\right) \longleftarrow A \longleftarrow 0 .
$$

The boundary maps are $\partial_{j}^{\vee}:=\operatorname{Hom}_{A}\left(\partial_{j}, A\right)$, which send $\prod_{i=1}^{z_{j-1}} A\left(s_{i}^{j-1}\right)$ into $\prod_{i=1}^{z_{j}} A_{>0}\left(s_{i}^{j}\right)$ for all $j$. By definition, $\operatorname{Ext}_{A}^{d}\left(k_{A}, A\right)=\prod_{i=1}^{z_{d}} A\left(s_{i}^{d}\right) / i m\left(\partial_{d}^{\vee}\right)$. Since the image $i m\left(\partial_{d}^{\vee}\right)$ is in $\prod_{i} A_{>0}\left(s_{i}^{d}\right), \operatorname{dim}_{k}\left(\operatorname{Ext}_{A}^{d}\left(k_{A}, A\right)\right) \geq z_{d}$. By hypothesis, $\operatorname{Ext}_{A}^{d}\left(k_{A}, A\right)$ is finite dimensional and hence $z_{d}$ is finite. We use downward induction to show that every $z_{j}$ is finite. Suppose $z_{j+1}$ is finite. If $z_{j}$ is infinite, then there is a finite free submodule $\oplus_{i=1}^{n} A\left(-s_{i}^{j}\right) \subset \oplus_{i=1}^{z_{j}} A\left(-s_{i}^{j}\right)$ such that the image of $\partial_{j+1}$ is in $\oplus_{i=1}^{n} A\left(-s_{i}^{j}\right)$. For every $i>n, i m\left(\partial_{j+1}\right) \cap A\left(-s_{i}^{j}\right)=0$ and $\partial_{j+1}^{\vee}\left(A\left(s_{i}^{j}\right)\right)=0$. Since $\operatorname{Ext}_{A}^{j}\left(k_{A}, A\right)=0, A\left(s_{i}^{j}\right) \subset \prod_{i} A\left(s_{i}^{j}\right)$ is in the image of $\partial_{j}^{\vee}$. But the image of $\partial_{j}^{\vee}$ is in $\prod_{i} A_{>0}\left(s_{i}^{j}\right)$, a contradiction. Therefore every $z_{j}$ is finite. Next we show that

$$
\max \left\{s_{i}^{j} \mid 1 \leq i \leq z_{j}\right\}<\max \left\{s_{i}^{j+1} \mid 1 \leq i \leq z_{j+1}\right\}
$$

for all $j$. If not, there is an integer $i_{0}$ such that $s_{i_{0}}^{j} \geq s_{t}^{j+1}$ for all $t$. Since (3-1) is minimal, $\partial_{j+1}$ sends the generator of $A\left(-s_{i}^{j+1}\right)$ into $\oplus_{i \neq i_{0}} A\left(-s_{i}^{j}\right)$, whence 
$i m\left(\partial_{j+1}\right) \subset \oplus_{i \neq i_{0}} A\left(-s_{i}^{j}\right)$. Hence $\partial_{j+1}^{\vee}\left(A\left(s_{i_{0}}^{j}\right)\right)=0$ and $A\left(s_{i_{0}}^{j}\right)$ is in the image of $\partial_{j}^{\vee}$. But the image of $\partial_{j}^{\vee}$ is in $\oplus_{i} A_{>0}\left(s_{i}^{j}\right)$, a contradiction. Therefore (3-4) holds.

Let ${ }_{A} F$ be the finite dimensional left $A$-module $\operatorname{Ext}^{d}\left(k_{A}, A\right)$. Then (3-3) is a free resolution of ${ }_{A} F$. Since $\underline{\operatorname{Hom}}(\underline{\operatorname{Hom}}(P, A), A)=P$ for all finitely generated free modules $P, \underline{\operatorname{Ext}}^{d}\left({ }_{A} F, A\right)=k$ and $\underline{\operatorname{Ext}}^{i}\left({ }_{A} F, A\right)=0$ for all $i \neq d$. Since $F$ is finite dimensional, by [Zh, 1.1], $\underline{\operatorname{Ext}}^{i}\left({ }_{A} k, A\right)=0$ for all $i \neq d$. Let $w=\operatorname{dim}_{k}\left(\underline{\operatorname{Ext}}^{d}\left({ }_{A} k, A\right)\right)$. Then $1=\operatorname{dim}_{k}\left(\operatorname{Ext}^{d}(F, A)\right)=w \operatorname{dim}_{k}(F)$. Hence $\operatorname{dim}_{k}(F)=w=1$. Therefore $F=k(e)$ and $\underline{\operatorname{Ext}}^{d}\left({ }_{A} k, A\right)=\underline{\operatorname{Ext}}^{d}(F, A)(e)=k(e)$. Thus we have proven part 1 and part 2 .

Since $\operatorname{dim}_{k}\left(\underline{\operatorname{Ext}}^{d}\left(k_{A}, A\right)\right) \geq z_{d}$, we have $z_{d}=1$, and the fact that $\underline{\operatorname{Ext}}^{d}\left(k_{A}, A\right)=$ $A\left(s_{1}^{d}\right) / i m\left(\partial_{d}^{\vee}\right)$ shows that $s_{1}^{d}=e$. Hence the $d$-th term of the minimal free resolution of $k_{A}$ is $A(-e)$ and (3-4) shows that $0<s_{i}^{j}<e$ for all $0<j<d$ and all $i$. Therefore part 3 follows.

Since the $i$-th term of the minimal free resolution of $k_{A}$ (respectively ${ }_{A} k$ ) is isomorphic to $\underline{\operatorname{Tor}}_{i}^{A}\left(k_{A},{ }_{A} k\right) \otimes_{k} A$ (respectively $\left.A \otimes_{k} \underline{\operatorname{Tor}}_{i}^{A}\left(k_{A},{ }_{A} k\right)\right), k_{A}$ and ${ }_{A} k$ have the same type of minimal free resolutions. Consequently, $c_{k_{A}}(t)=c_{A} k(t)$. By (3-3), $\pm t^{l} p_{A}\left(t^{-1}\right)$ is the characteristic polynomial of ${ }_{A} k$. Hence $\pm t^{l} p_{A}\left(t^{-1}\right)=p_{A}(t)$ where $l=\operatorname{deg}\left(p_{A}(t)\right)$. Since (3-1) is the minimal free resolution of $k_{A}$ and (3-3) is the minimal free resolution of ${ }_{A} k(e), c_{k(e)}(t)=(-1)^{d} p_{A}\left(t^{-1}\right)$. Hence $e=l=\operatorname{deg}\left(p_{A}(t)\right)$ and $t^{l} p_{A}\left(t^{-1}\right)=(-1)^{d} p_{A}(t)$. By either (3-2) or (3-4), $d \leq e$. Therefore part 4 follows.

If $d=e$, by (3-2) and (3-4), $\min \left\{s_{i}^{j} \mid 1 \leq i \leq z_{j}\right\}=\max \left\{s_{i}^{j} \mid 1 \leq i \leq z_{j}\right\}=j$. Hence part 5 follows. Finally, by Corollary 2.2, $\operatorname{GKdim}(A) \leq m\left(p_{A}(t)\right) \leq e$, proving part 6 .

As an immediate consequence of Proposition 3.1.1, we have the following.

Corollary 3.2. If $A$ is a commutative connected graded regular algebra, then $A$ is noetherian.

Remark. 1. [SmZ] generalized Corollary 3.2 to the following: every commutative connected graded ring with global dimension $d$ is isomorphic to the polynomial ring $k\left[x_{1}, \ldots, x_{d}\right]$ with $\operatorname{deg}\left(x_{i}\right)>0$.

2. We will see in Example 3.4 that there is a connected graded finitely presented regular ring of global dimension 2 which is not noetherian. Hence we cannot delete the hypothesis that $A$ is commutative from Corollary 3.2 .

3. It is unknown if we can replace 'commutative' by 'PI' in Corollary 3.2.

There are two well-known families of connected graded noetherian algebras of global dimension 2. Let $a, b, c, d \in k$ such that $a d-b c \neq 0$. Let $r_{a, b, c, d}$ denote the element $a x^{2}+b y x+c x y+d y^{2}$ in the free algebra $k\langle x, y\rangle$. Then $k\langle x, y\rangle /\left(r_{a, b, c, d}\right)$ is a regular noetherian domain of global dimension 2. In this case $\operatorname{deg}(x)=\operatorname{deg}(y)>0$.

Another family is $k[x][y ; \sigma, \delta]$ where $\sigma$ is a graded algebra automorphism of $k[x]$ and $\delta$ is a graded $\sigma$-derivation. Then $R=k[x][y ; \sigma, \delta]$ is a regular noetherian domain of global dimension 2. In this case $\operatorname{deg}(y)>\operatorname{deg}(x)>0$. (We could also have $\operatorname{deg}(x)=\operatorname{deg}(y)$ in this case, but then $R$ would be isomorphic to an algebra of the form $k\langle x, y\rangle /\left(r_{a, b, c, d}\right)$.)

We will prove that there are no other connected graded noetherian algebras of global dimension 2. First we show that there are no other regular algebras of global dimension 2 with finite GK-dimension. This is well known ([Ste, Lemma 2.2.5] gives 
the result when $k$ is algebraically closed). For completeness, we include a proof in the general case.

Proposition 3.3. Let $A$ be a connected graded regular algebra of global dimension 2. If $A$ has finite GK-dimension, then $A$ is isomorphic to either

(a) $k\langle x, y\rangle /\left(r_{a, b, c, d}\right)$ where $a d-b c \neq 0$, or

(b) $k[x][y ; \sigma, \delta]$ where $\sigma$ is an automorphism of $k[x]$ and $\delta$ is a $\sigma$-derivation.

Proof. By Proposition 3.1, we have an augmented minimal free resolution of $k$

$$
0 \longrightarrow A(-e) \longrightarrow \oplus_{i=1}^{z} A\left(-s_{i}\right) \longrightarrow A \longrightarrow k \longrightarrow 0
$$

where $1<s_{i}<e$ for all $i$. Hence $h_{A}(t)=p_{A}(t)^{-1}=\left(1-\sum_{i=1}^{z} t^{s_{i}}+t^{e}\right)^{-1}$. Since $\operatorname{GKdim}(A)<\infty$, by Lemma 2.1, every root of $p_{A}(t)$ has absolute value 1 . By the Intermediate Value Theorem, $p(1) \geq 0$ and hence $z \leq 2$. Since $t^{e} p\left(t^{-1}\right)=p(t)$, $p(t)=1-t^{i}+t^{2 i}$ or $p(t)=1-t^{i}-t^{j}+t^{i+j}$. But $\left(1-t^{i}+t^{2 i}\right)^{-1}=1+t^{i}-t^{3 i}+\cdots$, which cannot be a Hilbert series. Hence $p(t)=1-t^{i}-t^{j}+t^{i+j}$, and thus the augmented minimal free resolution of $k_{A}$ is

$$
0 \longrightarrow A(-(i+j)) \longrightarrow A(-i) \oplus A(-j) \longrightarrow A \longrightarrow k \longrightarrow 0 .
$$

As a consequence, $A$ is generated by two elements $x$ and $y$, with $\operatorname{deg}(x)=i$ and $\operatorname{deg}(y)=j$, subject to one relation in degree $i+j$. Let the relation be $y_{1} x+x_{1} y=0$ where $\operatorname{deg}\left(y_{1}\right)=j$ and $\operatorname{deg}\left(x_{1}\right)=i$. Then the boundary map from $A(-i) \oplus A(-j)$ to $A$ is $\partial_{1}(a, b)=x a+y b$ and the boundary map from $A(-(i+j))$ to $A(-i) \oplus A(-j)$ is $\partial_{2}(a)=\left(y_{1} a, x_{1} a\right)$. Since the dual of (3-5) is the minimal free resolution of ${ }_{A} k$, $A$ is generated by $x_{1}$ and $y_{1}$. If $i=j$, then $y_{1}=a x+b y$ and $x_{1}=c x+d y$ with $a d-b c \neq 0$. Then $A \cong k\langle x, y\rangle /\left(r_{a, b, c, d}\right)$. If $i<j$, then $x_{1}=a x$ and $y_{1}=c y+d x^{s}$ with $a c \neq 0$ (the term $d x^{s}$ appears only when $s=j / i$ is an integer). In this case, the relation is axy $=\left(c y+d x^{s}\right) x$ and $A \cong k[x][y ; \sigma, \delta]$ for some $\sigma$ and $\delta$.

Remark. If $k$ is assumed to be algebraically closed, we have $k\langle x, y\rangle /\left(r_{a, b, c, d}\right) \cong$ $k[x][y ; \sigma, \delta]$ some graded automorphism $\sigma$ of $k[x]$ and some $\sigma$-derivation $\delta$. Thus, in this case, any connected graded regular algebra of global dimension two with finite GK-dimension is isomorphic to an algebra of the form $k[x][y ; \sigma, \delta]$.

Now we turn to the case where $A$ is not assumed to have finite GK-dimension. By Proposition 3.1.3, if $A$ is a regular algebra of global dimension 1, then $A$ is isomorphic to $k[x]$. If $A$ is regular of global dimension 2, then $A$ is generated by two or more elements subject to one relation. Proposition 3.3 shows that $A$ has finite GK-dimension if and only if $A$ is generated by two elements. The next example shows that there exist regular algebras of global dimension two generated by more than two elements. Such rings are non-noetherian and have exponential growth.

Example 3.4. There is a non-noetherian connected graded regular algebra of global dimension 2 .

Let $R=k\langle x, y, z\rangle /\left(x^{2}+y^{2}+z^{2}\right)$. First we prove that $\operatorname{gl} \operatorname{dim}(R)=2$. Let $F$ be the free algebra $k\langle x, y, z\rangle$ and let $b=x^{2}+y^{2}+z^{2} \in F$. Let $S$ be the idealizer $\mathbb{I}(b F):=\{f \in F \mid f b \in b F\}$. It is routine to check that $S=k+b F$. Hence the eigenring (defined in $[\mathrm{Di}, 2.1]$ ) is $E=S / b F \cong k$. By [Di, 5.3], gl. $\operatorname{dim}(R)=$ gl. $\operatorname{dim}(E)+2=2$. Now consider the augmented minimal free resolution of the 
trivial module $k$

$$
0 \longrightarrow R(-2) \longrightarrow R(-1)^{\oplus 3} \longrightarrow R \longrightarrow k_{R} \longrightarrow 0 .
$$

The boundary map $\partial_{1}$ from $R(-1)^{\oplus 3}$ to $R$ is defined by $\partial(a, b, c)=x a+y b+z c$ and the boundary map $\partial_{2}$ from $R(-2)$ to $R(-1)^{\oplus 3}$ is defined by $\partial_{2}(a)=(x a, y a, z a)$. Applying $\underline{\operatorname{Hom}}(-, R)$ to the free resolution of $k_{R}(3-6)$, we obtain

$$
0 \longleftarrow R(2) \longleftarrow R(1)^{\oplus 3} \longleftarrow R \longleftarrow 0
$$

where the boundary maps are $\partial_{2}^{\vee}$ and $\partial_{1}^{\vee}$. By direct computation, $\partial_{2}^{\vee}(a, b, c)=$ $a x+b y+c z$ and $\partial_{1}^{\vee}(a)=(a x, a y, a z)$. Therefore (3-7) is the minimal free resolution of ${ }_{R} k(2)$. Thus $\underline{\operatorname{Ext}}^{i}\left(k_{R}, R\right)=0$ for $i \neq 2$ and $\underline{\operatorname{Ext}}^{2}\left(k_{R}, R\right)=k(2)$, showing that $R$ is regular. By $(3-6), h_{R}(t)=\left(1-3 t+t^{2}\right)^{-1}$ and this implies that $R$ has exponential growth. By Theorem 1.2, $R$ is non-noetherian.

Remark. 1. A classification of connected graded regular rings of global dimension two was given in [SmZ]. A connected graded ring is regular of global dimension two if and only if it is isomorphic to $k\left\langle x_{1}, \ldots x_{n}\right\rangle /\left(r_{\sigma}\right)$, where (i) $n \geq 2$, (ii) $1 \leq$ $\operatorname{deg}\left(x_{1}\right) \leq \ldots \leq \operatorname{deg}\left(x_{n}\right)$, (iii) $\operatorname{deg}\left(x_{i}\right)+\operatorname{deg}\left(x_{n-i}\right)$ is a constant for all $i$, (iv) $\sigma$ is a graded algebra automorphism of the free algebra $k\left\langle x_{1}, \ldots x_{n}\right\rangle$, and (v) $r_{\sigma}=$ $\sum_{i=1}^{n} x_{i} \sigma\left(x_{n-i}\right)$. For example, let $\operatorname{deg}\left(x_{i}\right)=1$ and $\sigma\left(x_{i}\right)=x_{n-i}$ for all $i$, then $k\left\langle x_{1}, \ldots, x_{n}\right\rangle /\left(\sum_{i=1}^{n} x_{i}^{2}\right)$ is regular of global dimension two.

2. By adding central indeterminates, we can easily construct non-noetherian regular algebras of arbitrarily large global dimension. In particular, there are non-noetherian regular rings of global dimension three. Therefore the condition $\operatorname{GKdim}(A)<\infty$ is necessary in the classification of 3 -dimensional regular rings in [AS] [ATV].

Finally we prove Theorem 0.4 from the introduction.

Theorem 3.5. 1. If $A$ is a connected graded noetherian algebra of global dimension 2, then $A$ is regular and $\operatorname{GKdim}(A)=2$. Moreover, and $A$ is isomorphic to either

(a) $k\langle x, y\rangle /\left(r_{a, b, c, d}\right)$ where $a d-b c \neq 0$, or

(b) $k[x][y ; \sigma, \delta]$ where $\sigma$ is an automorphism of $k[x]$ and $\delta$ is a $\sigma$-derivation.

Proof. By Theorem 2.4.1, $A$ has finite GK-dimension. By Proposition 3.3, it suffices to prove that $A$ is regular. By [ATV, 3.15], $A$ is a domain (note that, in the proof of $[\mathrm{ATV}, 3.15]$, the regularity hypothesis was not used). By [SZ, (3.8.1)], for any finitely generated right $A$-module $M$ there is a convergent spectral sequence

$$
E_{2}^{p, q}:=\underline{\operatorname{Ext}}_{A}^{p}\left(\underline{\operatorname{Ext}}_{A}^{q}(M, A), A\right) \quad \Longrightarrow \quad \mathbb{H}^{p-q}(M),
$$

where $\mathbb{H}^{p-q}(M)=0$ if $p \neq q$ and $\mathbb{H}^{0}(M)=M$. The $E_{2}$-page of (3-8) (that is, the table of $E_{2}^{p, q}$ terms) is

$$
\begin{array}{lll}
\mathrm{E}^{0,2}(M) & \mathrm{E}^{1,2}(M) & \mathrm{E}^{2,2}(M) \\
\mathrm{E}^{0,1}(M) & \mathrm{E}^{1,1}(M) & \mathrm{E}^{2,1}(M) \\
\mathrm{E}^{0,0}(M) & \mathrm{E}^{1,0}(M) & \mathrm{E}^{2,0}(M)
\end{array}
$$

where $\mathrm{E}^{p, q}(M)$ denotes $\operatorname{Ext}_{A}^{p}\left(\operatorname{Ext}_{A}^{q}(M, A), A\right)$. The boundary maps in the $E_{r^{-}}$ page have degree $(r, r-1)$. Hence the terms $\mathrm{E}^{1,2}(M), \mathrm{E}^{0,2}(M), \mathrm{E}^{2,0}(M)$, and 
$\mathrm{E}^{1,0}(M)$ will survive in the $E_{\infty}$-page. Since these are off the main diagonal and (3-8) converges,

$$
\mathrm{E}^{1,2}(M)=\mathrm{E}^{0,2}(M)=\mathrm{E}^{2,0}(M)=\mathrm{E}^{1,0}(M)=0 .
$$

Let $Q$ be the quotient division ring of $A$. If $\mathrm{E}^{0,1}(M)=\underline{\operatorname{Hom}}_{A}\left(\underline{\operatorname{Ext}}_{A}^{1}(M, A), A\right) \neq 0$, then $\underline{\operatorname{Ext}}_{A}^{1}(M, A)$ is not torsion, which implies $Q \otimes \underline{\operatorname{Ext}}_{A}^{1}(M, A) \neq 0$. But by [SZ, $3.3]$,

$$
Q \otimes \underline{\operatorname{Ext}}_{A}^{1}(M, A)=\operatorname{Ext}_{Q}^{1}(M \otimes Q, Q)=0 .
$$

This is a contradiction and hence $\mathrm{E}^{0,1}(M)=0$. Now let $M=k$. Since $A$ is a domain and infinite dimensional, $\mathrm{E}^{0,0}(k)=0$. Since (3-8) converges, $\mathrm{E}^{2,1}(k)$ is an image of $\mathrm{E}^{0,0}(k)$, and hence it is zero. Since $A$ has global dimension 2 , $\operatorname{Ext}_{A}^{2}(k, A) \neq 0$. But $\mathrm{E}^{1,2}(k)=\mathrm{E}^{0,2}(k)=0$, whence $\mathrm{E}^{2,2}(k) \neq 0$. Since $k$ is a simple module, from (3-8) we obtain $\mathrm{E}^{1,1}(k)=0$ and $\mathrm{E}^{2,2}(k)=k$. Therefore $\operatorname{Ext}_{A}^{i}(k, A)=0$ for all $i \neq 2$. By Corollary $2.5, \underline{\operatorname{Ext}}_{A}^{2}(k, A) \cong k(l)$ for some $l$ and hence $A$ is regular.

\section{ACKNOWLEDGMENT}

The authors would like to thank L. W. Small and S. P. Smith for several discussions on the subject.

\section{REFERENCES}

[AS] M. Artin and W. Schelter, Graded Algebras of Dimension 3, Adv. Math. 66 (1987), 172216. MR 88k:16003

[ATV] M. Artin, J. Tate and M. Van Den Bergh, Modules over Regular Algebras of Dimension 3, Inventions Math., vol. 106, 1991, pp. (335-389). MR 93e:16055

$[\mathrm{CF}]$ J. W. S. Cassels and A. Fröhlich, Eds., Algebraic Number Theory, Proceedings of an Instructional Conference, Academic Press, 1967. MR 35:6500

[Di] W. Dicks, On the cohomology of one-relator associative algebras, J. Alg. 97 (1985), 79-100. MR 87h:16041

[KL] G. Krause and T. H. Lenagan, Growth of algebras and Gelfand-Kirillov dimension, Research Notes in Mathematics, Pitman Adv. Publ. Program, vol. 116 (1985). MR 86g:16001

$[\mathrm{MR}]$ J. C. McConnell and J. C. Robson, Non-Commutative Noetherian Rings, WileyInterscience, Chichester, 1987. MR 89j:16023

[MS] J. C. McConnell and J. T. Stafford, Gelfand-Kirillov dimension and associated graded rings, J. Algebra 125 (1989), 197-214. MR 90i:16002

[NV] C. Năstăsescu and F. Van Oystaeyen, Graded Ring Theory, North Holland, Amsterdam, 1982. MR 84i: 16002

[RS] R. Resco and L. Small, Affine noetherian algebras and extensions of the base field, Bull. London Math. Soc. 25 (1993), 549-552. MR 94m:16029

[S1] M. K. Smith, Growth of twisted Laurent extensions, Duke Math. J. 49 (1982), 79-85. MR 83j: 16034

[S2] Universal enveloping algebras with sub-exponential but not polynomially bounded growth, Proc. Amer. Math. Soc. 60 (1976), 22-24. MR 54:7555

[SmZ] S. P. Smith and J. J. Zhang, Some non-noetherian regular graded rings, in preparation.

[SZ] J. T. Stafford and J. J. Zhang, Homological properties of (graded) noetherian PI rings, J. Algebra 168 (1994), 988-1026. MR 95h:16030

[St] R. P. Stanley, Generating Functions (G.-C. Rota, ed.), Studies in Combinatorics, Vol. 17, MAA Studies in Math., Washington: MAA, Inc., 1978, pp. (100-141). MR 81i:05015

[Ste] D. R. Stephenson, Artin-Schelter regular algebras of global dimension three, Ph.D. thesis, University of Michigan, UMI, 1994. 
[W] C. T. C. Wall, Generators and relation for the Steenrod algebra, Ann. Math. 72 (1960), 429-444. MR 22:7117

[Zh] J. J. Zhang, Connected graded Gorenstein algebras with enough normal elements, J. Algebra, to appear.

Department of Mathematics-0112, University of California at San Diego, La Jolla, California 92093-0112

E-mail address, D. R. Stephenson: dstephen@math.ucsd.edu

Department of Mathematics, Box 354350, University of Washington, Seattle, WashINGTON 98195

E-mail address, J. J. Zhang: zhang@math.washington.edu 